quelques produits français, ainsi que les noms de produits américains cités dans une étude de F. D. Goll (Milk Plant Monthly, t. XXV, no 2, février 1936 , p. 60).

\title{
RAPPORT D'UN TRAVAIL D'EXPÉRIMENTATION SUR LE CONTROLE DE LA IMAMIMITE STREPTOCOCCIQUE PAR LA VACCINATION CURATIVE ET PRÉVENTIVE ET QUELQUES MESURES HYGIÉNIQUES
}

par

\section{EDOUARD BROCHU}

$$
\text { L. S. A., }
$$

Ass-Professeur de Bactériologie

Institut Agricole d'Oka.

\section{Dr M. PANISSET}

M. V., Professeur de Bactériologio et de maladies contagieuses, Ecole de Médecine Vétérinaire de la province de Québec.

\section{Dr M. VEILLEUX}

M. V., Professeur de Pathologie Chirurgicale et de Clinique,

Ecole de Médecine Vétérinaire de la Province de Québec.

(Travail entrepris par la Société de Production animale du Comté des Deux-Montagnes.)

Nous tenons à témoigner notre reconnaissance à ceux qui ont rendu possible notre présent travail. Nos remerciments vont tout particulièrement :

A M. Gustave Toupin, professeur de Zootechnie, directeur de la Société de Production animale du Comté des Deux-Montagnes et organisateur du travail.

Au Ministère de l'Agriculture de la province de Québec pour son aide financière.

A l'Institut agricole d'Oka pour son aide matérielle.

Nos remerciements aussi au Dr J. M. Rosell, pour ses directives seientifiques, à MM. J. Perron, Ass-Professeur de Zootechnie, et E. PAGí, E. S. A., pour leur généreux concours, aux $\mathrm{D}^{\mathrm{r}}$ L. BÉRARD, bactériologiste, R. RAJOTTE, M. V., à M. O. Borsvert, M. Sc., au Prieuré Cistercien de Notre-Dame de Chicoutini pour leur contribution sous forme de rapports.

\section{PARTIE DOGUMENTAIRE}

\section{A. Préliminaires.}

De tout temps, les opinions sur la valeur des divers procédés thérapeutiques ont été partagées : " Hippocrate dit oui, mais Galien dit non. "Notre époque ne fait pas exception.

La science du criterium en thérapeutique ne semble pas à l'abri 
de tout doute. Des traitements donnent de bons résultats, selon certains médecins; d'autres n'y voient qu'insuccès. Ces faits un peu déconcertants peuvent trouver des explications différentes, dont voici quelques causes :

10 L'attribution très fréquente à l'intervention thérapeutique de guérisons qui seraient très probablement survenues naturellement.

$2^{\circ}$ La variation dans les circonstances ou les facteurs divers de l'expérience : caractère variable des maladies, surtout des maladies infectieuses; observation défectueuse tant dans l'appréciation du degré de maladie que du degré de guérison ; la race, la constitution de l'animal, le genre de nourriture, les états physiologiques différents, les maladies secondaires non perceptibles.

3o Trop souvent, la critique mal fondée ou mal intentionnée du résultat des traitements, faite par des observateurs étrangers à l'expérience.

$4^{\circ}$ La nature du traitement lui-même, souvent inégal malgré ses apparences : dosage différent, produit de qualité différente ou inférieure.

Ce dernier cas est surtout à envisager chez les divers expérimentateurs de la vaccination antimammiteuse. Pendant que les uns employaient des vaccins frais, autogènes, spécifiques, en quantité suffisante, et à intervalles répétés, et rapportaient des résultats favorables, d'autres ont utilisé des doses de vaccin incroyablement petites, d'un vaccin de qualité souvent douteuse, et obtenaient des résultats négatifs.

Avant de rapporter nos propres résultats, nous croyons utile de résumer quelques-unes des communications présentées par d'autres investigateurs, qui ont aussi employé la vaccination comme mesure thérapeutique ou prophylactique, avec le double objectif de guérir et de prévenir et dont les résultats paraissent plutôt encourageants pour l'usage de la vaccination.

\section{B. Résultats obtenus antérieurement.}

Du livre "Revue de littérature sur la mammite bovine jusqu'à la fin de 1933 ", vol. II, pages 233-242, par E. Munch Peterseen, M. Sc., Ph. D. et F. D. McMasteur, Laboratoire d'hygiène animale, Section d'Hygiène animale, Université de Sydney, nous reproduisons textuellement les extraits suivants : (1)

“ REID [1] procéda à des injections sous-cutanées dans le flane, aux doses de $0 \mathrm{~cm}^{3} 5$ à $2 \mathrm{~cm}^{3}$ avec un vaccin autogène à $10-12$ jours d'intervalle. Le vaccin était préparé avec une culture sur bouillon âgée de deux jours de Str. pyogenes qui avait été tué par chauffage

(1) Traduction C. WoLf. 
à $58^{\circ} \mathrm{C}$. pendant une heure. Le traitement fut entièrement couronné de suceès.

"Von Ostertag et Weichel [2] ont indiqué qu'il leur fut impossible d'immuniser eontre un type inhabituel de mammite qui était très répandu en Allemagne à ce moment et donnait des eultures pures virulentes per os, ou par injections sous-cutanées de cultures vivantes ou tuées de $B$. pyogenes, alors que des injections intra-veineuses donnaient une immunité parfaite, immunité qui n'était, toutefois, que de courte durée.

"PAYne [3] essaya un vaccin personnel, composé de streptocoques et staphylocoques tués, par injections sous-eutanées dans le pli de la peau au-dessus du pis sur trois vaches. Chaque vache reçut en tout 4 injections de $1 \mathrm{~cm}^{3}$, en commençant par une dose de 250 millions d'organismes et en terminant par 750 millions, à intervalles de trois jours. Toutes furent guéries.

"Carpenter [4] a décrit des expériences avec injections de vaccins autogènes vivants ou tués. Sur 40 eas traités avec le vaccin autogène, six seulement $(15 \%)$ ne furent pas entièrement guéris.

"HenRy [5] a traité plusieurs troupeaux avee un vaccin spécifique préparé par les Laboratoires Sérothéraphiques du Gouvernement australien contre la mammite streptococcique. La dose était de $10 \mathrm{~cm}^{3}$ et 10 autres centimètres eubes après 6 jours. Si aucune amélioration n'avait lieu, on injectait encore $10 \mathrm{~cm}^{3}$. En tout, environ 150 vaches, la plupart très infectées, furent traitées de cette façon, et il affirma que de très bons résultats furent obtenus. Le vaccin était particulièrement bon à titre prophylactique, mais probablement pas aussi bon au point de vue curatif ; mais on admit que, de toutes façons, il évitait l'expansion de la maladiè.

"Albiston [6] éprouva la valeur des vaccins sur un troupeau se composant de 95 animaux, dont 38 étaient atteints de mammite streptococcique ; trois des animaux étaient atteints très dangereusement d'infections complexes (streptocoques et staphylocoques). Le nombre des vaches atteintes atteignit 42 avant que la vaccination ne fût commencée. Le troupeau fut divisé en deux groupes : le premier étant traité avec un vaccin autogène; le second, avec le vaccin préparé par les Laboratoires Sérothéraphiques du Gouvernement australien. Les deux vaccins employés avaient à peu près la même force en ce qui concerne le nombre d'organismes par centimètre cube, et quatre injections furent effectuées à chaque vache avec les mêmes séries de doses. Le troupeau fut ré-examiné 25 jours après l'administration de la dernière dose avec les résultats suivants : sur six animaux $(14 \%)$, plus aucune trace de mammite streptococcique, tandis que $26(86 \%)$ étaient encore infectés. Sur les six animaux, deux avaient été traités avec le vaccin autogène, et quatre 
avec celui du Laboratoire Sérothéraphique du Gouvernement. 26 de ces vaches souffraient de mammite chronique avec un peu d'induration de la glande au moment du traitement.

"STICKORN [7] réussit à guérir 7 animaux sur 8 avec un vaccin polyvalent. Dans un autre troupeau, tous les animaux injectés, sauf un, furent guéris, et le reste fut considéré comme protégé. II essaya aussi des vaccins autogènes sur 17 troupeaux. Il affirma que l'immunité était de durée variable, allant de 3 à 9 mois. Il ne rencontra jamais de blessure ou de lésion causées par l'application du vaccin, même aux doses de $100 \mathrm{~cm}^{3}$.

"Lentz [8] utilisa comme vaccin une culture atténuée de streptocoques cultivés sur agar, puis enlevés par lavage. Il pratiqua des injections sous-eutanées du vaccin avec $10 \mathrm{~cm}^{3}$ à 10 et 14 jours d'intervalle. Dans quelques cas, les animaux furent isolés du troupeau, et quelques-uns traits à fond à intervalles rapprochés en complément de la vaccination. Il indiqua que, dans la plupart des cas, une injection amena la guérison, surtout dans les plus récents, mais dans les cas chroniques les animaux furent vaccinés une seconde fois après 10 à 14 jours. Jamais il ne pratiqua de troisième injection. On ne nota pour ainsi dire pas de réapparition de la maladie.

"Turner [9] indiqua que l'utilisation de bactérine streptococcique avant parturition avait été couronnée d'un plein succès et avait empêché le développement des pis distendus, phénomène qui a souvent lieu quand il y a mammite. Il traita 39 vaches de cette façon, et celles-ci vêlèrent sans former de pis distendus. Sur 24 cas de mammite à divers degrés, il donna une moyenne de 6 doses de bactérine et obtint 18 guérisons.

"Un anonyme [10] indiqua que 11.806 vaches de 250 troupeaux d'importance différente et de parties diverses de la NouvelleZélande furent vaceinées avec un vaccin bien connu d'origine privée pendant les années 1925-1926 et 1926-1927. Les propriétaires indiquèrent que 4.416 de ces vaches avaient de la mammite avant que la vaccination n'eut lieu. Lors de la saison suivant la vaccination, le nombre de vaches atteintes dans les mêmes fermes était de $\mathbf{1 . 4 1 4}$ (environ 3.000 étant guéries).

"K Ковцосн [11] conseilla l'utilisation de l' "autopyothérapie ", qui ne causa aucun trouble. On pourrait l'appeler un auto-vaccin très fort (potenzierte Autovakzine); elle consistait en 2 ou 3 séries de 3 injections, en doses de $0 \mathrm{~cm}^{3} 01$ à $0 \mathrm{~cm}^{3} 02$ de la propre sécrétion purulente du pis de la vache. Il traita 105 eas, tous avec succès.

"GLieten BeRG [12] obtint d'excellents résultats par des injections de culture sur bouillon (cultivée à $37^{\circ} \mathrm{C}$. pendant 24 heures) de diplocoques dans les cas d'infections diplococciques.

"Rosell [13] fit des essais de vaccination sur 104 vaches mam- 
miteuses de sept troupeaux différents. Le vaccin était préparé comme suit : les races caractéristiques isolées furent cultivées sur viande de cheval-glucose et bouillon-lactose avec infusion de bouillon de cœur qui étaient les milieux produisant le plus grand nombre de bactéries par centimètre cube $\left(44\right.$ heures à $37^{\circ} \mathrm{C}$. dans des flacons d'Erlenmeyer d'un demi-litre). On ajoutait souvent du carbonate de calcium stérile. On utilisait habituellement la culture entière sur bouillon. Pour les premières injections, la culture fut chauffée pendant 25 minutes à $58^{\circ} \mathrm{C}$. et $0,4 \%$ de phén ’l furent ajoutés. Ultérieurement, on n'utilisa que des suspensions bactériennes vivantes, et on ajoutait de temps en temps co me agent atténuateur et conservateur $0,2-0,3 \%$ de phénol aux vaccins vivants et non chauffés. Le vaccin était administré sous forme d'injection intra-musculaire à intervalles allant de 5 à 8 jours ; les doses progressives suivantes étant employées : $15 \mathrm{~cm}^{3} 30$ à $40 \mathrm{~cm}^{3}$ de vaccin dans les deux premières périodes ; 40 à $80 \mathrm{~cm}^{3}$ dans les dernières vaccinations. Dans les deux premières injections, on n'employa que des vaccins chauffés, et dans les autres, des vaccins vivants à la dose de 25 à $60 \mathrm{~cm}^{3}$. Ces séries d'injections furent répétées après deux mois si les vaches continuaient à montrer des symptômes de mammite et aussi une troisième fois, deux mois après, si nécessaire. Dans tous les cas, en même temps que les suspensions bactériennes, on injectait aussi 40 à $60 \mathrm{~cm}^{3}$ de lait pasteurisé à $60^{\circ}$ pendant une demi-heure. Dans d'autres cas, du lait mammiteux, auquel on avait ajouté 4 gouttes de formol commercial par $1.000 \mathrm{~cm}^{3}$ de lait fut injecté, le formol ayant été préalablement dissous dans quelques centimètres cubes d'eau. Sur environ 1.600 injections opérées, on observa seulement dans cinq cas une formation semblable à une tumeur avec réactions inflammatoires locales.

"Le nombre total de vaches utilisé pour essayer l'effet curatif du vaccin fut de 104, le nombre total de quartiers malades étant de 265. Le nombre de quartiers qui, 10 à 15 jours après les premières séries d'injections, était, d'après les symptômes cliniques, amélioré, fut de 209. Après une seconde période de vaccination, le nombre de quartiers améliorés était de 224. Quelques vaches ayant été abattues, et d'autres ayant vêlé, ou étant taries, ne purent pas être examinées après la deuxième ou troisième période de vaccination. Sur les 104 vaches traitées, 64 furent considérées comme guéries de la mammite, au point de vue symptômes, dans tous leurs quartiers après une série de vaccinations deux mois plus tard: 71. Sur le total de 104 vaches traitées, 71 vaches furent considérées comme guéries. Après le traitement de la mammite, on constata une augmentation de la production de lait dans un troupeau de 55 vaches. En tout, 
$78 \%$ des quartiers malades furent guéris, ou $68,21 \%$ des vaches atteintes.

«Vaccination préventive : cette expérience fut effectuée sur un troupeau de 83 vaches. Deux injections (de 15 à $25 \mathrm{~cm}^{3}$ de vaccin chauffé et de 10 à $25 \mathrm{~cm}^{3}$ de vaccin vivant) furent pratiquées avec un intervalle de huit jours. Ces vaches n'accusaient aucun symptôme de mammite un ou deux mois avant la vaccination ; mais avaient été conservées dans des troupeaux contenant des vaches infectées. Deux mois plus tard, un examen montra que 64 ou 69 vaches étaient normales, et que trois avaient un à deux quartiers avec de légers symptômes de mammite, mais on ne put obtenir de streptocoques pour eulture que de deux de ces dernières. Dans un autre troupeau de dix vaches vaccinées saines, deux eurent de la mammite après très peu de temps.

"Kinssig et Argermann [14] affirment que l'utilisation de vaccins dans le traitement de la mammite n'a pas donné de bons résultats, alors qu'utilisés d'une façon prophylactique, ils ont donné des résultats prometteurs.

“ Des injections prophylactiques furent exécutées sur 308 vaches dans six troupeaux. Aucun nouveau cas ne se produisit pendant les six semaines ayant suivi les injections dans $89,6 \%$ des cas; mais 32 vaches $(10,1 \%)$ furent infectées malgré les injections, et sur ces 32 vaches, $19(6,2 \%)$ donnèrent des signes cliniques de mammite certaine et $13(4,2 \%)$ donnèrent des streptocoques. Il est possible, toutefois, que ces vaches étaient déjà porteuses de streptocoques avant l'injection, quoiqu'aucun organisme ne fut trouvé dans le lait examiné. "

Dans le même livre bibliographique, page 233, on rapporte que les Auteurs dont les noms suivent affirment aussi avoir obtenu de bons résultats avec la vaccination et tous la recommandent. Ce sont : Frei [15a], Perl [17], Stader [18], Schmoker [19], Huynen [20], Ferguson [21], SchlaAk [22], Moore [23], Pfeiler, Sohlaak et Thomseb [24], Sachweh [25], Supfle et Hofmann [26], Pokorny [27], Richter et Demmel [28], Schuann [29], Graub et Zsонокке [30], Michigan Agricultural Experiment Station [33], Dykstra [34], Krage et Gipman [35], O. Gion [36].

Ceux dont les noms suivent rapportent que la vaccination peut prévenir les manifestations aiguës de la mammite : REID [37], Proscholdt [38], Ranbiger [39], Cory [40], Pound [41], Anonyme [42], Fethers [43], Hindmarsh [44], Kiessig [45] et Brigham, MCALPINE et ANDERSON.

Du livre " De la mammite chez les vaches ", par M. E. Whalley Section des Informations, Conseil national des Recherches, Ottawa, juillet 1932, nous transcrivons les extraits suivants (1):

(1) Traduction C. WoLf, 
(Dans l'expérience de la Section du bétail du Ministère de l'Agriculture, New-South. Wales [47], la seule méthode de traitement de la mammite contagieuse chez les vaches laitières, qui ait donné des résultats satisfaisants, fut la vaccination avec un vaccin préparé avec les organismes causes de la maladie. L'opinion créée à la suite de cé travail exécuté sur 12 troupeaux (à peu près 500 vaches) est qu'on pourrait compter sur le vaccin pour protéger le bétail des attaques de cette maladie, étant entendu que les animaux ne soient pas infectés au moment de la vaccination. La valeur curative du vaccin a semblé être variable et les fermiers furent prévenus de n'avoir à vacciner que les vaches saines, à titre de mesure préventive. Cette méthode a donné d'excellents résultats.

"CORY [48] indique dans son rapport que les traitements, à la fois préventifs et curatifs, ont été couronnés de succès à l'aide de vaccins préparés à la Station d'Essais de bétail, Yeerongpilly, Queensland. Quand il est employé à titre préventif, le vacein confère une période d'immunité contre la mammite contagieuse, variant suivant les individus, mais n'excédant pas douze mois. L'époque la plus favorable pour employer le vaccin à titre préventif est celle précédant ou suivant immédiatement le vêlage, c'est-àdire quand la vache est habituellement plus susceptible d'être malade.

"Un vaccin "spécifique " peut être utile à titre curatif, mais les meilleurs résultats sont habituellement obtenus avec un vaccin autogène, e'est-à-dire avec un vaccin préparé avec la race particulière de microbe affectant les animaux qu'on se propose de traiter. Le vaccin est injecté avec une seringue à injection dans les tissus sous-cutanés mous derrière l'épaule. La dose complète de vaccin dans les cas ordinaires est de $4 \mathrm{~cm}^{3}$ injectés en deux doses de $2 \mathrm{~cm}^{3}$ chaque avec intervalle de 48 heures entre les injections. Deux injections suffisent habituellement pour la guérison, mais dans les cas anciens, il peut être nécessaire de continuer le traitement.

“ Carpenter [49] de l'Université Cornell a effectué, récemment, une étude complète de l'utilisation des suspensions vivantes de streptocoques alpha-hémolytiques pour le contrôle de la mammite bovine. En résumant les résultats de son travail, il indique que l'injection de ces organismes semble donner une certaine protection à la vache contre la maladie. Il est intéressant de noter que, sur les 12 cas de mammite qui se déclarèrent sur 132 animaux traités, presque tous eurent lieu dans le premier ou le deuxième mois suivant les injections de streptocoques. Ceci peut indiquer qu'une période de 30 ou 60 jours est nécessaire pour donner une immunité, qui sera suffisamment importante pour protéger les vaches contre e invasion des streptocoques et de l'inflammation en résultant. 
"En utilisant une préparation faite avec des suspensions autogènes de streptocoques morts et vivants isolés d'échantillons de lait: mammiteux, CARPENTEr a trouvé que quoique les injections de streptocoques morts fussent de valeur certaine, des résultats bien meilleurs furent obtenus en employant des suspensions vivantes. de cet organisme; ainsi, ce dernier type diminua la durée de la. maladie et arrêta l'inflammation subséquente, de sorte qu'il n'y eut que peu de modifications de tissus.

"BRyan, C. S., 1933, "Etudes sur les vaccins dans la mammite infectieuse ", Journ. Amer. Vt. Med. Assoc., 83, N. SL., 36, 390-399, et dans un Bulletin de la Station d'Essais agricoles du Michigan ; et Bryan, C. S., MM. Burlingame et E. B. Cavell, 1933 "Nouvelles Etudes sur le lacto-vaccin dans la mammite, Vet. Med,, 28, $398-400$ [50] indiquent que les cultures vivantes de streptocoques. obtenues du troupeau atteint, en traitement, furent d'une valeur: appréciable pour le traitement des animaux malades et qu'elles. protégèrent les animaux non atteints. Un " lacto-vaccin » consistant en un lait additionné de streptocoques auquel était ajouté du violet de gentiane à la concentration de 1 pour 10.000 fut aussi considéré comme ayant une eertaine valeur. Malheureusement, on $\mathbf{n}$ 'utilisa pas d'animaux de contrôle, et les animaux ne furent en observation que pendant peu de temps; il est donc impossible d'évaluer exactement l'influence des vaccins utilisés par BRYAN et ses collaborateurs. sur la mammite dans les troupeaux qu'ils traitèrent.

"Dans une autre étude qui vient d'être publiée par le "Bulletin trimestriel de la Station d'Essais agricoles du Michigan », vol. 17, $n^{\circ}$ 4, mai 1935 : "Valeur du lacto-vaccin pour la protection des vaches non malades dans un troupeau atteint de mammite ", BRYAN expose sa conclusion de la façon suivante :

"L'administration du lacto-vaccin aux vaches non atteintes: dans un troupeau atteint de mammite réduit l'extension de l'infection et protège, dans beaucoup de cas, toutes les vaches, non atteintes par l'infection, qui existent dans le troupeau. Si le programmed'extirpation de la maladie s'étend sur une période de plusieurs: années, il est convenable de vacciner les animaux non infectés au moins une fois par an. Les génisses qui n'ont pas encore donné de lait ou qui n'ont pas de lésion sur le pis peuvent en toute sécurité être achetées ou élevées comme animaux de remplacement. Toutefois, elles devront être vaccinées avant d'être saillies. Il est désirable d'éliminer les vaches infectées aussitôt que possible pour diminuer les sources de streptocoques infectieux de la mammite. ")

Nous donnons pour finir quelques extraits du travail de Otto. GIon, de la Faculté de Médecine-Vétérinaire de l'Université deGiessen, Allemagne. (Travail intitulé « Zur Therapie der Streptokok- 
kenmastitis ", Giessen, 1932). Cet Auteur apporte les conclusions suivantes : $a$ ) les essais d'immunisation préventive des vaches en lactation par des vaccins streptococciques préparés avec des germes du troupeau sous traitement, a donné de bons résultats ; $b$ ) cette même vaccination préventive tentée sur des vaches en dehors de l'état de gestation a donné moins de résultat que chez les vaches en gestation ; $c$ ) les effets curatifs de ces vaccins dans les cas aigus n'ont pas été satisfaisants ; d) la même vaccination dans les cas chroniques a donné de meilleurs résultats que dans les cas aigus.

De ce même travail publié par l'Institut de Santé vétérinaire de Bavière, nous transcrivons encore quelques extraits :

"WILD [51] rapporte avoir obtenu la disparition des streptocoques des vaches malades avec une seule injection d'un vaccin préparé avec 11 souches différentes de Strept. agalactiae.

"SUPFLE et HoFMANN [52] ont traité avec succès la mammite de trois troupeaux avec le vaccin autogène. Strckdorn [53] consigne avoir obtenu de bons succès curatifs dans de grands troupeaux de vaches malades, traitées par le vaccin spécifique autogène, ou par l'introduction intraveineuse de sérum. Il recommande spécialement le vaccin. Il conseille de n'utiliser le sérum que pour les vaches manifestant une fièvre élevée ou atteintes de maladie aiguë.

"Goetze [54] conseille l'usage mixte de vaccins et de chimio ou protéino-thérapie.

“LENTz [55] conseille également les traitements par injections de vaccins autogènes à des intervalles de 10 à 14 jours, et cela tant pour traitement curatif que pour traitement préventif.

«Glietenberg [56] recommande d'introduire les traitements de vaccination tant préventifs que curatifs avec des matériaux virulents et spécifiques et traiter par cette vaccination tant la vache malade que la vache saine de toutes les étables. Comme mesure secondaire, il conseille la séparation des vaches malades, la traite fréquente, au moins quatre fois par jour, et l'observation des règles d'hygiène convenables, particulièrement la désinfection.

"SchWARZ [57] rapporte les résultats du traitement de 116 vaches par injection avec des cultures spécifiques de l'étable, appliquées à huit reprises différentes. Il affirme avoir obtenu la guérison de 105 de ces 116 vaches malades et conseille tout spécialement la séparation des animaux malades et l'examen répété des nouvelles vaches à introduire dans le troupeau.

"Rudolf. [58], qui ne concède pas à la vaccination une grande valeur curative, conseille néanmoins l'usage des vaccins spécifiques de l'étable pour empêcher la transmission de la maladie aux animaux sains.

"Krake et Gipmann [59] eroient qu'à l'époque actuelle, on ne 
peut obtenir de bons résultats qu'avec la combinaison de vaccinations spécifiques autogènes et de mesures hygiéniques.

"ZURECK [60] recommande comme le seul moyen existant dans la lutte contre la mammite infectieuse, la vaccination avec des vaccins spécifiques autogènes et la traite fréquente. "

Nous transcrivons du bulletin "La mammite streptococcique de la vache " par J. M. RoseLL, les citations suivantes :

( STICKDoRN, directeur de l'Institut Vétérinaire de Landberg, (Tierarz, Edsh., 434, 1936), croit que la thérapeutique vaccinale dans la mammite n'a pas été appréciée jusqu'à cette date, comme elle aurait dû l'être. Il déclare d'après ses expériences de vaccination, au moyen de vaccins polyvalents et de vaccins avee des cultures de la même étable, avoir obtenu les plus excellents résultats, tant dans la guérison des vaches malades que pour empêcher la propagation de la maladie d'une vache à l'autre, dans des troupeaux infectés. Selon lui, on réussit non seulement à arrêter la maladie, mais aussi à stimuler de telle façon la formation d'anticorps dans les animaux malades, que même les lésions arrivent à leur guérison. Il dit encore qu'il parvenait à protéger contre l'infection toutes les vaches vaccinées. La durée de l'état d'immunisation, après la vaccination, n'est pas égale chez toutes les vaches. Dans la plupart, la résistance à l'infection, obtenue par la vaccination, a duré plus d'une année et chez d'autres vaches, moins de temps.

"SUPFle et Hofmann (Munch, Tierarzt, Wochenschrift, 14, 1927) et Lentz, Berliner (Tierartz, Wochenschrift, 42, 1927), émettent un jugement très favorable sur la thérapeutique vaccinale. Avec des streptocoques spécifiques de l'étable, ils sont arrivés à détourner rapidement la maladie et à déterminer la guérison sans aucun autre traitement. De la même façon, ils ont évité avec des vaccinations préventives l'infection des vaches saines.

"LENTz, dans un rapport sur 400 vaches vaccinées, indique avoir eu la guérison d'un grand nombre de vaches avec une seule injection, spécialement quand il s'agissait de vaches où la maladie n'existait que depuis peu de temps. Il ajoute que les cas de récidive chez les vaches guéries devenaient plus rares.

"Wilk (Deutsch, Tierarzt, Wochschr., p. 154, 1926) déclare avoir eu $67 \%$ de guérisons au moyen de la vaccination chez ses vaches malades.

"RUDOLF, directeur de l'Institut d'Hygiène Vétérinaire de Vienne (Munch, Tierarzt, Wochschr., no 5, 1929), conseille le traitement vaccinal surtout dans les cas récents. Sur dix vaches, parmi celles qui, au point de vue clinique, microscopique et biologique, étaient complètement guéries quinze jours après la vaccination, on pouvait trouver encore des streptocoques après six mois, et sans. 
aucune manifestation de la maladie. En effet, chez quatre de ces vaches, six mois plus tard, on trouva encore les streptocoques, mais aucun signe de la maladie. Ceci démontre bien que les vaches atteintes de la mammite, en quelques circonstances, peuvent guérir de la lésion, tout en restant porteuses de streptocoques. En même temps, ceci nous montre que l'on ne doit pas considérer comme synonyme la présence du streptocoque et l'existence de la mammite. Un petit nombre d'auteurs, SEELEMANN entre autres, se sont basés presque exclusivement sur la présence du streptocoque dans le lait pour juger de l'effet de la vaccination. "

Sur ce point, il serait intéressant de citer les observations de Glimtenberg (Zeitschr, fur Fleisch und Milch-Hygiene, 1929, p. 158), qui, dans des travaux basés sur plus de 3.000 examens, croit que le streptocoque de la mammite peut exister dans des quartiers normaux sous forme de diplocoques, la plupart du temps phagocytés, et sans pouvoir pathogène, pouvant être cultivés et se présenter alors en chaînes, observations que nous avons aussi faites bien des fois. La présence des streptocoques en chaînes dans le lait, selon GLietenberG, serait un symptôme du manque de réaction d'immunité et, dans ce cas, le streptocoque changerait son chimiotactisme négatif de l'état de diplocoque en chimiotactisme positif dans lequel le streptocoque serait en chaînes.

GraU B et Zschокке (Schweitz, Arc., Tierheilk., 1929, p. 399) rapportent de très bons suecès obtenus en Suisse avec le traitement des vaccins autogènes. Il affirme aussi avoir obtenu une immunisation d'une durée de 6 à 9 mois par la vaccination préventive.

SACHWEH (Deutsch. Tierarzt. Wochschr., 1927, p. 777) rapporte de bons succès par le traitement vaccinal qu'il conseille de pratiquer quelques semaines avant le tarissement de la vache, ou pendant son état sec.

Gotze (Berl. Tierarzt. Wochschr., 1928, p. 381) n'obtient que très peu de résultats avec une seule injection de vaccin. Il considère qu'il est nécessaire de faire au moins trois ou cinq injections répétées.

Même alors, chez plusieurs vaches, prétend-il, les streptocoques étaient encore décelables dans le lait après la vaccination, bien que le lait fût redevenu normal dans ses caractères et que les symptômes cliniques fussent disparus. Il ajoute que dans beaucoup de ses vaches mammiteuses traitées par le vaccin, les modules inflammatoires ont disparu. Selon lui, on ne peut s'empêcher de reconnaître l'effet curatif de la vaccination dans la mammite, quand ces vaccinations sont faites d'une façon systématique et persistante.

Kingman ( N. Amer. Vet., 1929, no 10, 1928) considère la vaccination comme d'une utilité spéciale pour empêcher la propagation 
de la mammite. Kiessig (Deutsch. Tierarzt. Wochschr., 1929, p. 676) en dit autant.

SEDDON (Congrès Int. de Méd. de Londres, 1930, p. 286) rapporte qu'il n'obtint en Australie aucun bon résultat par le traitement pharmacothérapeutique, tandis que les résultats étaient variables avec le traitement par vaccins. Il pratiqua extensivement la vaccination préventive et obtint en général de bons résultats. Il conseille de vacciner, si possible, tout le troupeau avant le vêlage, faisant deux injections à quinze jours d'intervalle. Quand bien même les animaux vaccinés ne résisteraient pas à l'infection expérimentale, les résultats obtenus sur-le-champ chez un grand nombre d'animaux, indiquent que la vaccination préventive tend à procurer une résistance considérable chez les animaux vaccinés. Cette résistance est manifestée aussi par le fait que les cas de maladie qui se présentent chez les animaux vaccinés sont beaucoup plus bénins.

Krage et HiPMAN, de l'Institut bactériologique de la Chambre agricole de la Silésie (Archiv. für Tierheilkunde, 1931), rapportent, d'après leurs essais de vaccinations thérapeutiques et prophylactiques faits sur un nombre restreint de vaches, les conclusions suivantes : quelques vaches fortement infectées furent guéries et les streptocoques disparurent du lait : pour d'autres vaches, la guérison fut partielle et la présence du streptocoque fut encore décelable. Quoique les résultats ne puissent pas toujours être considérés comme bons, on ne peut cependant méconnaître l'action bienfaisante des vaccins tant thérapeutiques que prophylactiques. Il a été considéré, en général, que les vaccins curatifs amélioraient la maladie dans la plupart des cas, tandis que la production laitière augmentait visiblement. De la même façon que le traitement hygiénique et d'autres ne suffisent pas pour guérir la maladie, le traitement vaccinal doit être aidé du traitement hygiénique, surtout de la mulsion fréquente et complète. Les deux traitements ensemble donnent le meilleur résultat.

On ne peut toujours juger de la guérison, exclusivement par la présence ou l'absence des streptocoques, mais par la disparition des symptômes de la maladie et le retour de la production du lait à la normale.

Dans la vaccination prophylactique, il paraît être démontré que par cette vaccination préventive, la glande mammaire devient plus résistante contre l'infection et celle-ci se présente plus faible et souvent de très courte durée chez les vaches vaceinées.

Un fait digne d'observation, e'est que presque tous les auteurs qui ont annoncé avoir eu de bons résultats avec la vaccination ont utilisé des vaccins préparés avec les streptocoques cultivés, frais, du même troupeau sous traitement, ou, des vaceins préparés 
avec des souches autogènes et des souches étrangères formant ainsi un vaccin polyvalent, souvent renforcé par addition d'autres germes.

Le nombre d'investigateurs qui manifestent ne pas avoir de résultats par la vaccination est aussi relativement faible. Mais une lecture attentive des publications des auteurs qui ne sont pas partisans de la vaccination montre trop souvent que leur jugement n'est pas toujours basé sur une méthode scientifique ou sur un contrôle suffisant des résultats. Plusieurs de ces derniers expérimentateurs n'indiquent pas la dose de vaccin utilisée dans le traitement, ni l'origine ou la qualité des vaccins. D'autres, et ceux-là sont en grand nombre, ont utilisé des doses absolument insuffisantes, par exemple, 0,5 et 2 ou $5 \mathrm{~cm}^{3}$ de vaccin et souvent une seule fois. Il n'est pas nécessaire de commenter les succès qu'on pouvait attendre de ces traitements. D'autres ont utilisé les "vaccins stock » de laboratoires commerciaux, souvent envoyés d'autres pays. On sait ce que sont en général ces vaccins stock commerciaux. Ce sont des vaccins préparés parfois avec une seule souche d'une culture de laboratoire cultivée quelquefois même pendant des années dans ces laboratoires producteurs de vaccin. Même si ces vaccins stock sont préparés avec plusieurs sources de streptocoques, ils ont perdu depuis longtemps non seulement leur caractère virulent, susceptible de donner des réactions immunisantes suffisantes, mais souvent même, beaucoup de leurs caractères biologiques. Précisément, les streptocoques de la mammite, ainsi qu'il a été démontré aux laboratoires de l'Institut agricole d'Oka et par beaucoup d'institutions laitières de différents pays, sont des streptocoques qui changent très fréquemment leurs caractères quand ils sont cultivés en dehors de leur ambiance naturelle, qui probablement n'est autre que le pis de la vache. Par ailleurs, les vaccins commerciaux sont d'ordinaire d'une concentration insuffisante. On peut souvent observer, aussi, que les auteurs opposés à la vaccination recommandent généralement les traitements au moyen de quelques préparations chimiothérapiques patentées.

\section{Critique des communications au sujet de la vaccination, présentées jusqu'à cette date.}

D'après l'exposé que nous venons de faire sur les résultats de la vaccination obtenus jusqu'à ce jour, on peut voir qu'on ne s'est pas encore pleinement accordé sur la valeur de la vaccination ou préventive ou curative dans la mammite.

L'étude attentive de quelques-uns des travaux publiés nous amène, pour notre part, à conclure que les déductions tant de ceux qui rapportent de grands succès avee la vaccination (c'est la grande 
majorité) que de ceux qui, comme Semlemann et Diernhoffer, n'ont vu que des insuccès, peuvent être soumises à la critique.

A quelques-uns des premiers, on pourrait objecter d'avoir peutêtre proclamé les guérisons sans les avoir soumises à une assez longue période d'observation ou sans avoir toujours employé tous les procédés pouvant donner une garantie de eure pratiquement complète. Quant aux autres, qui nient la valeur de l'effet curatif du vaccin, ils paraissent appuyer leur jugement sur la présence du streptocoque dans le lait quelque temps après la vaccination. Se basant sur ce fait, ils nient la valeur de la vaccination sans prendre en considération la guérison clinique de la maladie. La présence du streptocoque dans le lait ne peut même pas être considérée comme le procédé le plus sûr pour montrer l'effet immunisant complet du traitement vaccinal. A côté de ce fait, on ne doit pas méconnaître, non plus, qu'en bien des cas, le processus inflammatoire qui constitue la maladie peut disparaître, ainsi que nous l'avons vérifié en plusieurs occasions, alors que le streptocoque persiste encore à l'intérieur de la mamelle. Ce fait est observé d'ailleurs dans toutes les maladies infectieuses, plus ou moins longtemps après la guérison et l'immunisation de l'individu. Les travaux de Steck ont surtout démóntré la grande ténacité de la mamelle à conserver des mois et même des années le type de la flore bactérienne qui s'y est une fois établi.

Théoriquement, on ne saurait guère attendre un effet immunisant décisif de la vaccination antistreptococcique, parce que cette bactérie ne montre pas, en général, dans l'antigénothérapie ou l'immunothérapie, un effet semblable à celui d'autres bactéries, par exemple, celles du groupe typhique, bipolaire, charbonneux, staphylococcique; les expériences sérologiques faites sur la séparation des groupes streptococciques à base des réactions d'immunisation, montrent néanmoins qu'on rencontre une réaction de nature défensive plus ou moins considérable dans les affections streptococciques.

On aurait une preuve décisive de la valeur immunisante streptococcique, si, dans l'emploi des vaccins, on évitait l'infection expérimentale du pis par le trayon. Mais les expériences faites jusqu'à cette date ne l'ont pas été d'une façon propreà donner de la sûreté. On est d'accord cependant à reconnaître, et notre expérience nous l'a aussi démontré, que l'infection streptococcique, la plupart du temps, est limitée à seulement un ou deux quartiers, bien moins souvent à trois quartiers, et assez rarement aux quatre quartiers.

Si l'on considère la grande facilité de propagation de la maladie d'une vache à l'autre, le fait de constater que l'infection ne se transporte pas d'un quartier malade à tous les autres quartiers, serait 
une raison pour nous encourager à croire que l'infection d'un quartier provoque un certain degré d'immunité dans les autres.

On doit aussi remarquer que, dans les essais de vaccination, il n'a pas pu encore être fixé de règles indiquant la quantité de germes nécessaires à introduire chez une vache, par voie sous-cutanée, pour obtenir un degré suffisant d'immunisation. La quantité de vaccin à injecter est bien différente chez les divers expérimentateurs. SemlemanN, par exemple, qui dans ses derniers travaux croit pouvoir tirer la conclusion que la vaccination est sans effet, a utilisé, dans ses expériences, des doses de vaccin de 5 à $20 \mathrm{~cm}^{3}$ d'une dilution de cultures en tubes d'agar, de $5 \mathrm{~cm}^{3}$ par tube, sans donner le nombre de germes par centimètre cube de son vaccin. Nos expériences nous ont montré que des injections de $300 \mathrm{~cm}^{3}$ de cultures vivantes, obtenues dans du bouillon avec sérum, foie, cœur et lactose, qui produit un nombre de germes bien plus grand que l'agar, n'ont donné aucune réaction perceptible sur les vaches chez qui nous les avons faites. En vaccinothérapie, nous nous approchons de la dose préventive et curative, quand celle-ci provoque les premières réactions thermiques ou d'augmentation de l'indice opsonique ou de réactions leucocytaires et autres.

Jamais on n'a pu fixer la dose de vaccin à donner à une vache pour la mammite, ni l'intervalle entre les doses. Bien que nous n'ayons pas eu l'occasion d'étudier ce point, comme nous désirions le faire, nous sommes bien loin de croire que les doses de 5 à $20 \mathrm{~cm}^{3}$ de suspensions de cultures d'agar, à intervalle de deux ou trois semaines entre chaque injection, comme SEELEMAN l'a fait, soient suffisantes ou se rapprochent de la dose nécessaire.

(A suivre).

\section{L'IMPORTANCE DU CUIVRE COMME MATÉRIAU POUR LA CONSTRUCTION DES APPAREILS EMPLOYÉS EN LAITERIE}

par

le Dr A. KARSTEN

Au cours de ces dernières années, il a été fait de grands "efforts pour remplacer le cuivre dans la construction des appareils électriques par d'autres métaux ou certains alliages, comme par exemple l'aluminium, certaines qualités d'aciers spéciaux, mais il semble que ces efforts n'aient pas donné de grands résultats.

Il y a quelques années, Mederer, de Munich, avait d'ailleurs publié une étude intéressante intitulée "Le cuivre en laiterie », dans laquelle cet Auteur souligne à juste titre que le cuivre reste toujours 